\title{
Rendimento comercial do tomateiro em resposta à salinização ocasionada pela fertigação em ambiente protegido ${ }^{1}$
}

\author{
W aleska M. Eloi' ${ }^{2}$, Sergio N. D uarte ${ }^{3}$, Tales M. Soares 4 , \\ Enio F. de F. Silva ${ }^{5} \&$ Jarbas H. Miranda ${ }^{6}$
}

\begin{abstract}
RESU MO
O objetivo deste trabalho foi avaliar o rendimento comercial da cultura do tomate em resposta a diferentes níveis de salinidade e manejo da fertigação sob ambiente protegido. 0 experimento foi conduzido em duas etapas, sendo a primeira desenvolvida no Laboratório de Solos e a segunda realizada em ambiente protegido, ambos localizados no Departamento de Engenharia de Biossistemas da ESALQ/USP, em Piracicaba. A primeira etapa consistiu de testes preliminares que possibilitaram a construção de curvas de salinização artificial, visando a salinização artificial do solo; na segunda etapa, conduziu-se a cultura utilizando-se seis níveis de salinidade inicial do solo e dois tipos de manejo. O bservou-se que o aumento da salinidade do solo reduziu o rendimento comercial da cultura. Para valores de condutividade elétrica do extrato de saturação (CEes) acima de $3,17 \mathrm{dS} \mathrm{m}^{-1}$, a produção relativa decresce $11,79 \%$ por aumento unitário da CEes. A cultivar de tomate Débora Plus apresentou maior tolerância à salinidade do que 0 encontrado na literatura $\left(2,5 \mathrm{dS} \mathrm{m}^{-1}\right)$.
\end{abstract}

Palavras-chave: manejo de nutrientes, salinidade limiar, Lycopersicum esculentum M ill

\section{Commercial yield of tomato in response to salinization caused by fertigation under greenhouse conditions}

\begin{abstract}
The objective of this study was to evaluate tomato crop and its commercial yield in response to different salinity levels and management of fertigation under greenhouse conditions. The experiment was conducted in two stages, being the first realized in the Laboratory of Soils and second accomplished in plastic greenhouse, both located in D epartment of Biosystems Engineering of ESALQ/U SP, in Piracicaba, Brazil. The first stage consisted of preliminary tests that made possible the construction of curves of soil salinization, for artificial salinization of the soil. In the second stage the effect of six levels of soil salinity and two types of crop management were studied. The increase of the soil salinity reduced the commercial crop yield. For values of electrical conductivity of the saturation extract over $3.17 \mathrm{dS} \mathrm{m}^{-1}$, production decreases by $11.79 \%$ per unit increase. "D ébora Plus" tomato presented higher tolerance to salinity than the results found in the literature $\left(2.5 \mathrm{dS} \mathrm{m}^{-1}\right)$.
\end{abstract}

Key words: nutrient management, salinity threshold, Lycopersicum esculentum Mill

Trabalho submetido e selecionado no primeiro Simpósio Brasileiro de Salinidade realizado de 12-15/10/2010 em Fortaleza, Ceará, Brasil ${ }^{1}$ Extraído da Tese do primeiro autor. Pesquisa financiada pelo CN Pq

${ }^{2}$ IFCE Campus de Sobral, Área de Recursos N aturais, Av. Dr. Guarany 317, Cidao, CEP 62040-730, Sobral, CE. Fone: (88) 3112-8100. E-mail: waleska@ifce.edu.br

${ }^{3}$ DER/ESALQ, Av. Pádua Dias, 11 CP: 9, CEP 13418-900, Piracicaba, SP. Fone: (19) 3447-8543. E-mail: snduarte@esalq.usp.br

${ }^{4}$ CCAAB/U FRB, Rua Rui Barbosa, 710, Centro, CEP 44380-000, Cruz das Almas, BA, Fone: (75) 3621-2798 E-mail: talesmiler@gmail.com

5 DTR/U FRPE, R. Dom Manoel de Medeiros, Dois Irmãos, CEP 52171-900, Recife, PE, Fone: (81) 3320-6261. E-mail: enio.silva@dtr.ufrpe.br

${ }^{6}$ DCE/ESALQ, Av. Pádua Dias, 11 CP: 9, CEP 13418-900, Piracicaba, SP. Fone: (19) 3429-4283. E-mail: jhmirand@esalq.usp.br 


\section{INTRODUÇÃO}

Dentre as hortaliças produzidas no Brasil o tomate se destaca como a principal, tanto no aspecto econômico quanto no social. O tomateiro é a segunda hortaliça cultivada no mundo, sendo sua quantidade produzida superada apenas pela batata (FAO, 2006). Goiás, São Paulo e Minas Gerais são os principais Estados produtores, respondendo por quase $70 \%$ da produção nacional. São Paulo responde por mais de $21 \%$ da produção nacional, em área de 11,6 mil ha (Nehmi et al., 2004). Entre as hortaliças mundialmente cultivadas para consumo in natura e, sobretudo, industrializados, o tomate se sobressai, razão por que é considerado de produção e utilização universal (Camargo et al., 2007).

A adição de fertilizantes via água de irrigação pode ocasionar a acumulação de sais dissolvidos na zona radicular, com a evapotranspiração das culturas, a qual remove a água e deixa a maior parte dos sais na superfície e em todo o perfil do solo. $\mathrm{O}$ uso de quantidades excessivas de sais solúveis acumulados na zona radicular das plantas dificulta a extração da solução salina do solo, resultando na redução do crescimento e da produtividade das plantas. A adição de fertilizantes via irrigação, sobretudo em ambiente protegido, tem elevado os níveis de salinidade do solo, chegando a ultrapassar os limites de tolerância das culturas, causando diminuição do rendimento ao longo de ciclos sucessivos (Dias et al., 2005; 2007). Queiroz et al. (2009) concluíram que a salinização em solos em ambiente protegido tende a agravar-se, em função da elevada proporção de produtores que não realizam monitoramento da condutividade elétrica do solo e da água.

O volume de sais adicionados ao solo via irrigação é proporcional ao volume de água aplicada, ou seja, a concentração de sais no solo cresce em função da lâmina de irrigação aplicada. Atualmente, a principal causa do acúmulo de sais em solos agrícolas tem sido as irrigações mal manejadas. O excesso de fertilização, o uso de água salina e a ausência de drenagem adequada são fatores que resultam em situações adversas que podem favorecer a degradação de solos (Silva et al., 2008).

Diversos autores citam os valores de salinidade limiar para várias culturas; entretanto, tais valores estão baseados no acúmulo de sais provenientes de irrigações com águas salinas. Neste contexto, Maas \& Hoffmann (1977) citam que o tomateiro tem um valor de salinidade limiar, expresso em termos de condutividade elétrica, de $2,5 \mathrm{dS} \mathrm{m}^{-1}$, reportando ainda que seu rendimento decresce para $50 \%$ quando a salinidade no extrato é de 7,6 $\mathrm{dS} \mathrm{m}^{-1}$, sendo classificado como cultura moderadamente sensível à salinidade. Eloi et al. (2007) comentaram sobre a necessidade de se determinar o grau de tolerância das culturas e monitorar a fertigação em ambiente protegido, visando ao controle da salinidade do solo.

A salinidade provocada por fertilizantes, e não por íons tóxicos, além do uso de ambiente protegido no cultivo do tomateiro devem promover diferenças em sua resposta à salinidade. De fato, nas referências tradicionais de tolerância das culturas à salinidade (Maas \& Hoffman, 1977) se ressalva que os valores apresentados devem ser considerados apenas como de tolerância relativa entre grupos de cultura, uma vez que valores de tolerância absoluta variam com o clima, condições de solo, tipo de sais e práticas culturais.

$\mathrm{O}$ fato de se cultivar à céu aberto ou em ambiente protegido pode levar a diferentes respostas das plantas à salinidade. Sonneveld (2004) mostra ser variável a resposta obtida sob sombreamento artificial ou sob diferentes estações do ano.

Medeiros et al. (2009) avaliaram a cultura do pepino, em ambiente protegido, conduzido sob condição salina, proveniente de sais fertilizantes, com seis níveis de salinidade do solo $\left(1,5 ; 2,5 ; 3,5 ; 4,5 ; 5,5\right.$ e $\left.6,5 \mathrm{dS} \mathrm{m}^{-1}\right)$ e verificaram que a produtividade máxima obtida foi $2,45 \mathrm{~kg} \mathrm{~m}^{-2}$ para o nível de salinidade $3,5 \mathrm{dS} \mathrm{m}^{-1}$; os mesmos autores encontraram o valor de 4,08 $\mathrm{dS} \mathrm{m}^{-1}$ para a salinidade limiar (SL) do pepino, com base na produção relativa da cultura.

Dias et al. (2005), com o objetivo de avaliar o uso de extratores de soluções do solo no auxílio ao manejo da fertigação e no controle da salinidade em solo cultivado com melão rendilhado, conduziram um estudo em ambiente protegido na cidade de Piracicaba, utilizando seis níveis de salinidade inicial do solo e dois manejos de fertigação: um tradicional e outro com controle da condutividade elétrica da solução do solo; os resultados mostraram que, com o uso de extratores, pode-se monitorar com precisão a concentração iônica da solução do solo. A salinidade do solo evoluiu com o tempo, estando os maiores níveis próximos à superfície do solo e do gotejador. A diferença no consumo de água entre os níveis de salinidade foi mais evidenciada no manejo controlado da fertigação, ajustando-se a um modelo quadrático.

O presente trabalho objetivou avaliar a produção comercial da cultura do tomate, cultivado em ambiente protegido, em resposta a diferentes níveis de salinidade e de manejo da fertigação.

\section{Material E MÉTODOS}

A metodologia utilizada foi adaptada de Silva (2002), sendo a primeira etapa do experimento desenvolvida no Laboratório de Solos e a segunda realizada em casa-de-vegetação plástica, localizada no Departamento de Engenharia Rural da ESALQ/ USP, situada em Piracicaba, São Paulo, nas coordenadas geográficas: $22^{\circ} 42^{\prime} \mathrm{S}, 47^{\circ} 38^{\prime} \mathrm{W}$, a uma altitude de $540 \mathrm{~m}$, no período entre 20 de novembro de 2006 e 09 de março de 2007.

A primeira etapa do experimento consistiu na realização de testes preliminares que possibilitaram a construção de curvas de salinização artificial, visando à realização do processo de salinização artificial do solo.

Para construção das curvas de salinização foi utilizada uma solução nutritiva, sugerida por Furlani et al. (1999). Em função do nível de condutividade elétrica desejada na solução, calculouse a quantidade de sais a se adicionar, mediante a Eq. 1 .

$$
\mathrm{C}=640 \mathrm{CE}_{\mathrm{s}}
$$

em que:
C - concentração de sais fertilizantes, $\mathrm{mg} \mathrm{L}^{-1}$
$\mathrm{CE}_{\mathrm{s}}$ - condutividade elétrica da solução preparada, $\mathrm{dS} \mathrm{m}^{-1}$ 
A partir de uma solução padrão com CE teórica de 10,5 dS m ${ }^{-1}$ e com equivalentes de $6,72 \mathrm{~g} \mathrm{~L}^{-1}$ de concentração salina, foram tomadas alíquotas de solução para cada nível de salinidade. Utilizou-se o total de 21 soluções. A concentração dessas soluções variou de 0 até $6,72 \mathrm{~g} \mathrm{~L}^{-1}$, com intervalos de $0,32 \mathrm{~g} \mathrm{~L}^{-1}$, o que corresponde, respectivamente, às salinidades teóricas variando de 0 até $10,5 \mathrm{dS} \mathrm{m}^{-1}$, com intervalos de $0,5 \mathrm{dS} \mathrm{m}^{-1}$, a partir da Eq. 1. A amostra em branco $\left(0 \mathrm{mg} \mathrm{L}^{-1}\right)$ correspondeu à condutividade elétrica inicial da água sem adição dos fertilizantes. A partir dessas amostras preparadas, determinouse a condutividade elétrica real das soluções, utilizando-se um condutivímetro.

A curva que relaciona a concentração de fertilizantes e a condutividade elétrica das soluções foi estabelecida por meio de um diagrama de dispersão, no qual foram plotados os valores da concentração de sais fertilizantes versus condutividade elétrica encontrada.

Posteriormente, amostras de solo foram coletadas, em seguida peneiradas, secas ao ar e acondicionadas em vasos de $20 \mathrm{~L}$, em cuja base perfurada tinha uma camada de envelope (brita + manta geotextil) de $2 \mathrm{~cm}$. O material de solo utilizado foi de um perfil classificado como Latossolo Vermelho fase arenosa, proveniente do campus da ESALQ e denominado Série "Sertãozinho".

Os níveis iniciais de salinidade do solo utilizados foram: $\mathrm{S}_{1}=1,5 \mathrm{dS} \mathrm{m}^{-1} ; \mathrm{S}_{2}=2,5 \mathrm{dS} \mathrm{m}^{-1} ; \mathrm{S}_{3}=3,5 \mathrm{dS} \mathrm{m}^{-1} ; \mathrm{S}_{4}=4,5 \mathrm{dS} \mathrm{m}^{-1}$; $\mathrm{S}_{5}=5,5 \mathrm{dS} \mathrm{m}^{-1} \mathrm{e} \mathrm{S}_{6}=6,5 \mathrm{dS} \mathrm{m}^{-1}$. A umidade do solo foi elevada até a máxima capacidade de retenção e, concomitantemente, foram adicionados os sais diluídos na água com o objetivo de se obter seis níveis de condutividade elétrica do extrato de saturação, baseados na curva de salinização, construída na primeira etapa. Três dias após a adição das diversas soluções salinas, amostras de solo foram retiradas dos vasos, nas camadas de 0 a $0,15 \mathrm{~m}$ e de 0,15 a $0,30 \mathrm{~m}$; a partir dessa amostragem, determinou-se a condutividade elétrica do extrato de saturação real, ou seja, aquela que corresponde aos valores encontrados no solo.

$\mathrm{Na}$ segunda etapa do experimento os tratamentos aplicados à cultura do tomateiro foram compostos pela combinação de dois fatores: salinidade inicial do solo com seis níveis $\left(\mathrm{S}_{1}=1,5\right.$ $\mathrm{dS} \mathrm{m}{ }^{-1} ; \mathrm{S}_{2}=2,5 \mathrm{dS} \mathrm{m}^{-1} ; \mathrm{S}_{3}=3,5 \mathrm{dS} \mathrm{m}^{-1} ; \mathrm{S}_{4}=4,5 \mathrm{dS} \mathrm{m}^{-1} ; \mathrm{S}_{5}=5,5$ $\mathrm{dS} \mathrm{m} \mathrm{m}^{-1} \mathrm{e} \mathrm{S}_{6}=6,5 \mathrm{dS} \mathrm{m}^{-1}$ ) e dois tipos de manejo de fertigação $\left(M_{1}\right.$ - de acordo com a marcha de absorção da cultura e $M_{2}$ com controle da condutividade elétrica da solução do solo).

Os 12 tratamentos propostos foram dispostos em 48 parcelas e o delineamento estatístico adotado foi o de blocos aleatorizados completos com quatro repetições, ficando os fatores estudados arranjados no esquema fatorial $6 \times 2$. Utilizouse a cultivar Débora Plus, cultivada em espaçamento de $0,50 \mathrm{~m}$ entre plantas e 1,20 m entre fileiras. $O$ transplantio das mudas foi realizado no dia 20 de novembro de 2006.

Adotou-se um sistema de irrigação por gotejamento, sendo o manejo baseado no monitoramento potencial mátrico da água no solo, obtido de tensiômetros instalados a $0,15 \mathrm{~m}$ de profundidade, e da curva característica de retenção de água no solo. A aplicação da irrigação foi realizada de acordo com a recomendação de Alvarenga (2004), sempre que a tensão encontrava-se entre 15 a $20 \mathrm{kPa}$. Aquantidade de água aplicada foi a suficiente para elevar a umidade à capacidade de campo, sendo calculada, mediante as médias das leituras tensiométricas, para cada tratamento.

A aplicação dos fertilizantes se deu via água de irrigação, uma vez por semana, sendo os tratamentos diferenciados em função dos manejos $M_{1}$ e $M_{2}$. O manejo $M_{1}$ baseou-se na marcha de absorção da cultura apresentada por Alvarenga (2004), o qual recomenda os seguintes valores totais para adubação de 1.000 plantas de tomateiro, via fertigação: $\mathrm{N}=35 \mathrm{~kg}, \mathrm{P}=14 \mathrm{~kg}$, $\mathrm{K}=75 \mathrm{~kg}$ e $\mathrm{Ca}=10 \mathrm{~kg}$. Para os tratamentos referentes ao manejo $\mathrm{M}_{2}$, realizou-se a fertigação objetivando-se manter os níveis iniciais de condutividade elétrica na solução do solo para cada tratamento. A quantidade de fertilizantes aplicada foi calculada para que a solução do solo se mantivesse no nível de CE inicial $\left(1,5 ; 2,5 ; 3,5 ; 4,5 ; 5,5\right.$ ou $\left.6,5 \mathrm{dS} \mathrm{m}^{-1}\right)$. Para este cálculo, utilizou-se a fórmula de mistura de soluções (Eq. 2).

$$
\mathrm{CE}_{\mathrm{c}}=\frac{\mathrm{V}_{\mathrm{cc}} \cdot \mathrm{CE}_{\mathrm{esd}}-\mathrm{V}_{\mathrm{a}} \cdot \mathrm{CE}_{\mathrm{a}}}{\mathrm{V}_{\mathrm{r}}}
$$

em que:

$\mathrm{V}_{\mathrm{cc}}$ - volume de água armazenado no solo à capacidade de campo, $\mathrm{L}$

$\mathrm{CE}_{\text {esd }}$ - condutividade elétrica do extrato de saturação do solo desejada, $\mathrm{dS} \mathrm{m} \mathrm{m}^{-1}$

$\mathrm{V}_{\mathrm{a}}$ - volume atual de água armazenado no solo, $\mathrm{dS} \mathrm{m}^{-1}$

$\mathrm{CE}_{\mathrm{a}}$ - condutividade elétrica atual do solo, $\mathrm{dS} \mathrm{m}^{-1}$

$\mathrm{V}_{\mathrm{r}}$ - volume de água no solo necessário para se atingir a capacidade de campo,L;

$\mathrm{CE}_{\mathrm{c}}$ - condutividade elétrica da solução de correção para se atingir a $\mathrm{CE}_{\text {esd }}, \mathrm{dS} \mathrm{m}^{-1}$

De acordo com o programado para o manejo $\mathrm{M}_{1}$, ou com o necessário para o manejo $\mathrm{M}_{2}$, os fertilizantes utilizados (nitrato de cálcio, nitrato de potássio, fosfato monoamônico, fosfato monopotássico, sulfato de potássio e sulfato de magnésio) foram diluídos nas águas de irrigação, correspondentes a cada tipo de manejo. A condutividade elétrica da solução foi medida após o término de cada evento de irrigação, utilizando-se, para extração da solução, cápsulas porosas sob vácuo.

Os valores de condutividade elétrica, determinada mediante a solução extraída pelas cápsulas porosas, foram corrigidos para a umidade de saturação, conforme sugerido por Silva (2002), Eq. 3.

$$
\mathrm{C}_{\text {estimada }} \mathrm{cp}=\frac{\mathrm{Ccp} \cdot \mathrm{Ucp}}{\mathrm{Us}}
$$

em que:

$\mathrm{C}_{\text {estimada }} \mathrm{cp}$ - condutividade elétrica ou concentração de íons no extrato de saturação, estimada a partir dos valores medidos na solução do solo obtida mediante extrator com cápsula porosa, $\mathrm{dS} \mathrm{m} \mathrm{m}^{-1}$ ou $\mathrm{mmol}_{\mathrm{c}} \mathrm{L}^{-1}$

Ccp - condutividade elétrica ou concentração de íons na solução do solo, obtida mediante extrator de cápsula porosa, $\mathrm{dS} \mathrm{m}{ }^{-1}$ ou mmol $\mathrm{L}^{-1}$ 
Ucp - umidade do solo no momento da retirada da solução com o extrator de cápsula porosa, $\mathrm{g} \mathrm{g}^{-1}$

Us - umidade do solo na pasta saturada, $\mathrm{g} \mathrm{g}^{-1}$

Após o início do período produtivo, avaliou-se a produção comercial de frutos $\left(\mathrm{kg} \mathrm{planta}^{-1}\right)$, considerando-se comerciais os frutos livres de danos mecânicos, manchas e deformações, e que atingiam o diâmetro transversal mínimo de $60 \mathrm{~mm}$; os resultados obtidos foram analisados estatisticamente.

A partir da Eq. 4, proposta por Maas \& Hoffman (1977), foi possível calcular a salinidade limiar (SL) da cultura do tomate.

$$
\mathrm{Y}=100-\mathrm{b}(\mathrm{CEes}-\mathrm{SL})
$$

em que:

$\mathrm{Y}$ - rendimento potencial, \%

CEes - salinidade do extrato de saturação, $\mathrm{dS} \mathrm{m}^{-1}$

SL - salinidade limiar da cultura, $\mathrm{dS} \mathrm{m}^{-1}$

b - diminuição do rendimento por aumento unitário de salinidade acima do valor $\mathrm{SL}, \%\left(\mathrm{dS} \mathrm{m}^{-1}\right)^{-1}$

\section{RESULTADOS E DISCUSSÃO}

Os resultados da curva de salinização artificial do solo permitiram relacionar a condutividade elétrica esperada e a observada e a concentração dos fertilizantes usados para sua construção (Figura 1A). Através da equação obtida por regressão linear, estimaram-se as quantidades de fertilizantes necessárias para se obter as condutividades elétricas desejadas. A equação obtida, qual seja, $\mathrm{CEs}=0,0008$. C+0,8443, diverge da originalmente proposta na Eq.1, em que a CEs = 0,00156.C, fato que, provavelmente, ocorreu em função da condutividade elétrica ser afetada pela valência e concentração relativa dos íons presentes na solução. A constante 0,8443 correspondeu à condutividade elétrica da água utilizada para o preparo da solução. Deve-se registrar que, apesar de muito usual em trabalhos envolvendo salinidade, a Eq.1 pressupõe condições específicas de tipos de sais dissolvidos, as quais não se repetem necessariamente em soluções de fertigação, como a proposta por Furlani et al. (1999).

Na Figura 1B são apresentados os resultados da salinização do solo, os quais apresentaram boa correlação entre os valores de CE estimados e observados no solo. Ressalta-se que a curva de salinização é específica para cada solo, dependendo, sobremaneira, das suas características físicas e químicas, dos fertilizantes e da adubação recomendada.

$\mathrm{Na}$ Figura 2A são mostrados os valores da condutividade elétrica da solução extraída nas cápsulas porosas instaladas a $15 \mathrm{~cm}$ de profundidade, ao longo do ciclo da cultura, para o manejo M1. Verificou-se um incremento nos níveis de salinidade do solo para os tratamentos S1, S2 e S3 e redução da salinidade entre os níveis mais elevados (S4, S5 e S6), com tendência ao equilíbrio no final.

De acordo com Dias et al. (2005), a tendência ao equilíbrio dos sais pode ser explicada pelo fato de que apenas parte dos sais incorporados através da fertigação fica no solo, pois parte é absorvida pela planta ou, ainda, torna-se insolúvel mediante

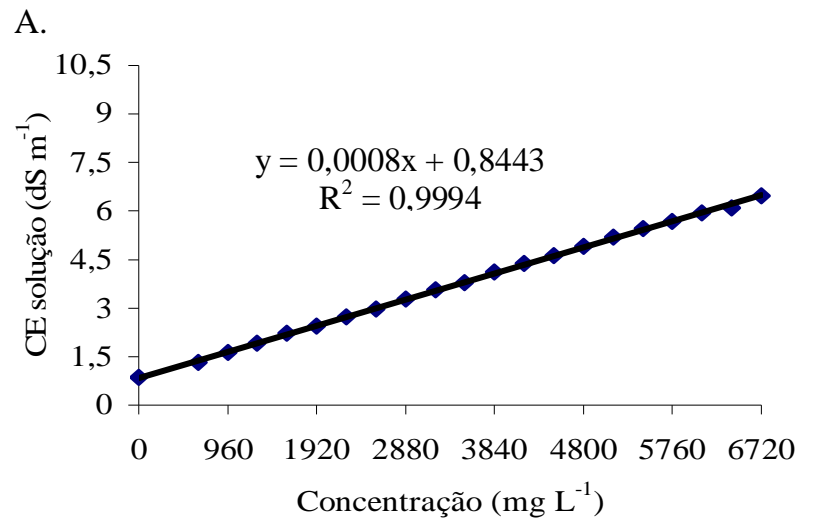

B.

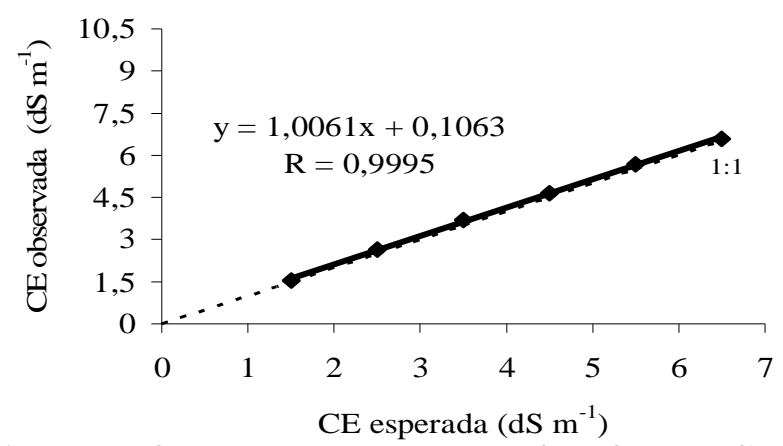

Figura 1. Relação entre a concentração das soluções salinas e a condutividade el étrica das soluções utilizadas (A) e CE esperada e a CE observada após a salinização do solo (B)

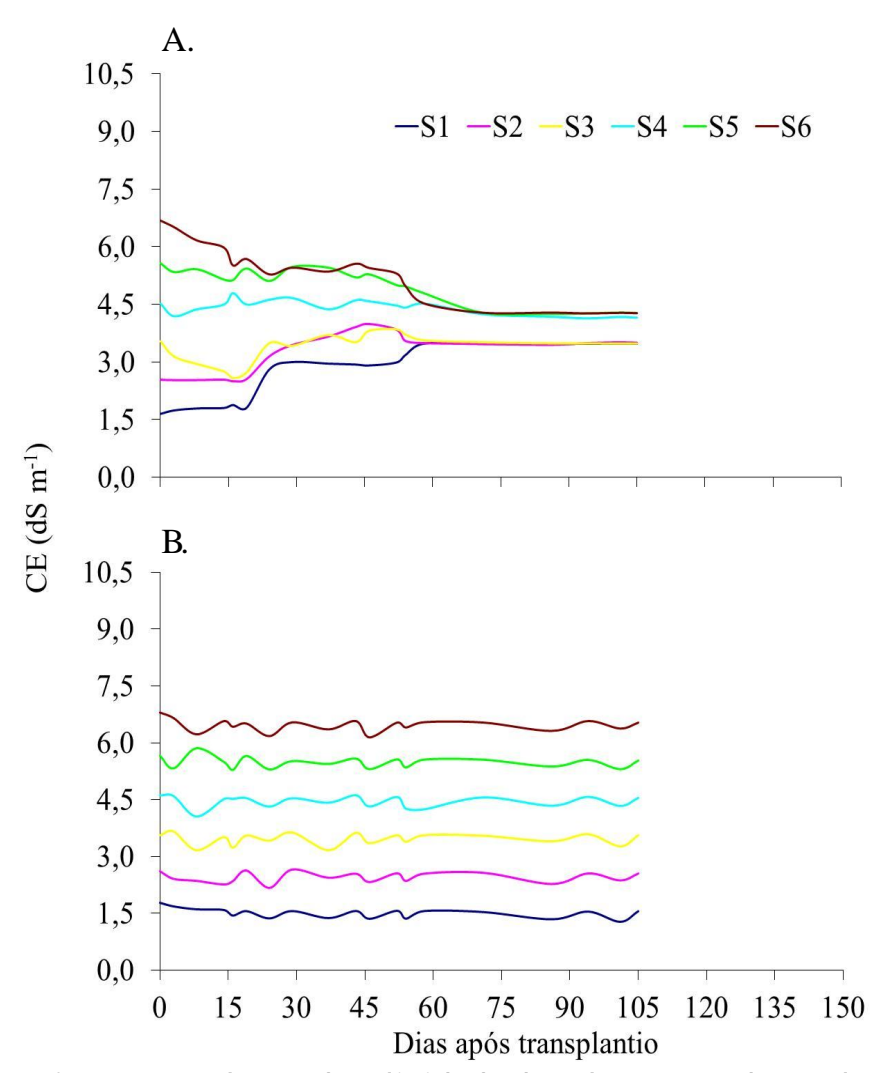

Figura 2. Evolução da salinidade do solo, para cada nível de salinidade inicial, ao longo do ciclo do tomate em função do manejo M 1 baseado na marcha de absorção (A) e do manejo M 2 com controle da condutividade elétrica do solo (B) 
a precipitação, quer por reações químicas ou por atingir limites de solubilidade na solução do solo.

Para o manejo com base na manutenção dos níveis de condutividade elétrica (Figura 2B), observa-se uma pequena variação nos valores da salinidade, indicando a eficiência do monitoramento e da correção da condutividade elétrica do solo. Este resultado está de acordo com o obtido por Silva (2002), que comentou que a salinidade do solo pode ser controlada a partir do conhecimento da salinidade do solo ao longo do ciclo da cultura e da correção das quantidades aplicadas de fertilizantes.

A análise de variância indicou que os diferentes níveis de salinidade influenciaram significativamente os resultados obtidos e que não houve interação entre manejo e os níveis de salinidade, evidenciando que o êxito que cada tratamento obteve não foi influenciado pelo manejo (Tabela 1). Esse resultado contraria a hipótese levantada, de maior rendimento na condição de controle do nível de salinidade do solo. Por outro lado, corrobora com os resultados encontrados por Silva (2002), que não observaram valores discrepantes entre os dois tipos de manejo empregados para a cultura do pepino. Um fator que se deve ponderar para a não comprovação da hipótese supracitada diz respeito à duração dos experimentos; em condições comerciais, cultivos sucessivos com ambos os tipos de manejo podem diferenciar os rendimentos encontrados ao potencializar os vários fatores negativos do aumento da salinidade, devido ao efeito osmótico crescente e ao acúmulo de íons específicos; por outro lado, no presente experimento, apesar do efeito osmótico causado pelo descontrole da salinidade ser de caráter mais imediato, os efeitos dos desequilíbrios nutricionais, da toxidez e das mudanças nas características físicas do solo não devem ser bem estabelecidos dentro do período de um único ciclo de cultivo.

Tabela 1. Resultados da análise de variância (quadrados médios) referentesao peso do fruto do tomateiro submetido a diferentes níveis de sal inidade e manejo da fertigação

\begin{tabular}{|c|c|c|}
\hline \multirow{2}{*}{ Fator } & \multicolumn{2}{|c|}{ Produção } \\
\hline & Total & Comercial \\
\hline Bloco & $26.189,33^{\mathrm{ns}}$ & $7.666,46^{\mathrm{ns}}$ \\
\hline Salinidade (S) & $858.262,41^{* *}$ & $803 \cdot 183,51^{* *}$ \\
\hline Manejo (M) & $143.807,46^{\mathrm{ns}}$ & $107.406,00^{\text {ns }}$ \\
\hline$S \times M$ & $68.760,32^{\mathrm{ns}}$ & $107.158,80^{\text {ns }}$ \\
\hline CV $(\%)$ & 18,15 & 20,18 \\
\hline
\end{tabular}

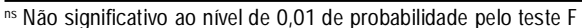

* Significativo ao nível de 0,05 de probabilidade pelo teste $F$

** Significativo ao nível de 0,01 de probabilidade pelo teste $F$

O rendimento máximo do tomateiro correspondente a $100 \%$ do rendimento comercial relativo foi de $2,06 \mathrm{~kg}_{\text {planta }}{ }^{-1}$. A produtividade média obtida foi inferior aos valores médios da cultura $64 \mathrm{Mg} \mathrm{ha}^{-1}$ (IBGE, 2009), o que pode estar associado às condições climáticas do período, pois a temperatura do ar e umidade relativa estavam acima do adequado para a cultura, como pode ser observado na Figura 3. As temperaturas mínimas variaram de 16 a $23^{\circ} \mathrm{C}$ e as máximas de 26 a $38^{\circ} \mathrm{C}$. De acordo com Alvarenga (2004), a faixa ótima é de 10 a $34^{\circ} \mathrm{C}$ e com relação à umidade relativa média foi de $75,7 \%$, a qual também se encontra acima da faixa ótima para a cultura que é de 50 a $70 \%$.

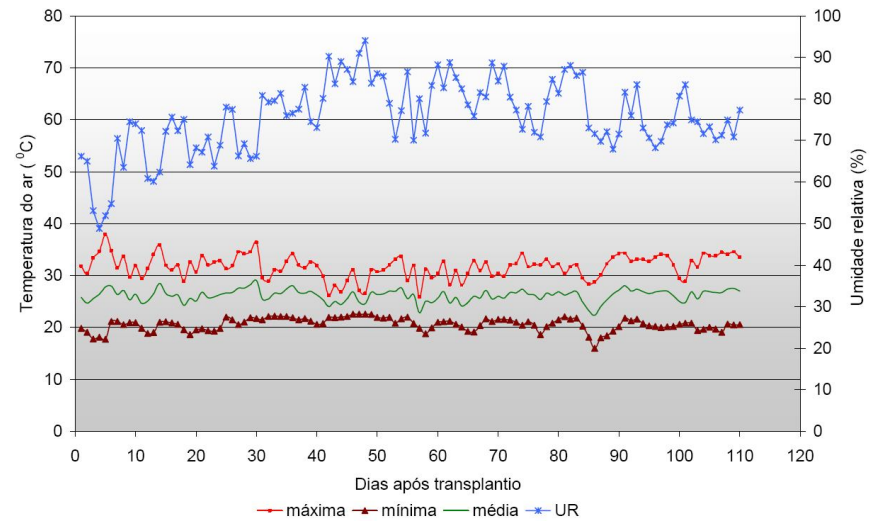

Figura 3. Temperaturas máxima, média e mínima e umidade relativa do ar ao longo do ciclo do tomateiro

A tolerância de várias culturas à salinidade é convencionalmente expressa em termos de produção relativa; verificou-se que a salinidade limiar (SL) do tomate cultivar Débora Plus foi de 3,17 dS m ${ }^{-1}$. Para valores de condutividade elétrica do extrato de saturação (CEes) acima de 3,17 dS m ${ }^{-1}$, a produção relativa decresceu $11,8 \%$ por aumento unitário da CEes (Figura 4).

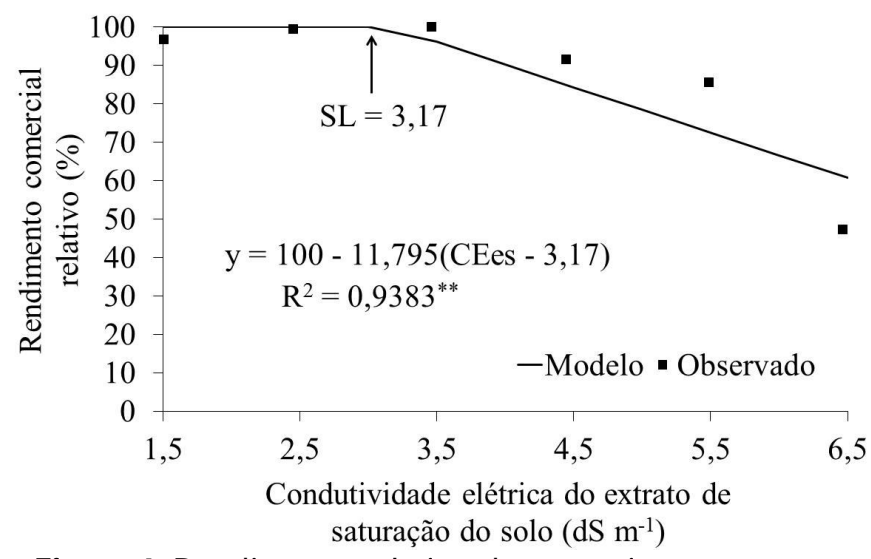

Figura 4. Rendimento relativo do tomateiro em resposta a diferentes níveis de salinidade do solo causada pela adição de fertilizantes

Maas \& Hoffmann (1977) informam, para o tomateiro, o valor de salinidade limiar, expresso em termos de condutividade elétrica do extrato de saturação, de $2,5 \mathrm{dS} \mathrm{m}^{-1}$ com decréscimo relativo de produtividade por unidade de CEes para a cultura de $9,0 \%$, sendo o valor encontrado por eles relativo ao uso de água salina. Saranga et al. (1991) definiram a salinidade limiar entre 2,0 e 2,5 dS m${ }^{-1}$ e que além desse valor há uma redução de 9 a $10 \%$ para cada acréscimo unitário da salinidade $\left(\mathrm{em} \mathrm{dS} \mathrm{m}^{-1}\right)$. Os valores encontrados por esses autores corroboram com os apresentados por Maas \& Hoffmann (1977). Por outro lado, em revisões de literatura mais atuais (Cuartero \& FernándezMuñoz, 1999), tem-se compendiado inúmeros trabalhos que demonstram o cultivo do tomateiro em condições de alta salinidade.

Trabalhando em condições hidropônicas no Sul do Brasil, Andriolo et al. (2003), concluíram ser possível reutilizar integralmente a solução nutritiva drenada no cultivo do 
tomateiro e que os efeitos da salinidade sobre a produtividade são observados somente com valores superiores a 4,9 dS m-1. Assim como no presente trabalho, Andriolo et al. (2003) avaliaram a salinidade provocada por fertilizantes e não por águas salobras com íons tóxicos.

O tomateiro é classificado como cultura moderadamente sensível à salinidade em relação ao uso de águas salinas (Maas \& Hoffmann, 1977); verificou-se, porém, que, em relação à salinidade provocada pelo uso dos fertilizantes, a cultura pode ser classificada como moderadamente tolerante à salinidade, nas condições estudadas. A mudança da classificação também pode ter ocorrido devido à cultivar utilizada, bem como pelo sistema e manejo da irrigação adotados. A irrigação por gotejamento reduz os efeitos da salinidade para a cultura, pois o solo se mantém mais úmido e os sais tendem a ser transportados para a periferia do bulbo úmido. Malash et al. (2008) comprovaram que a irrigação localizada promove maior crescimento e produção do tomateiro do que a irrigação por superfície, em condições salinas.

\section{ConclusõEs}

1. Não ocorreu diferença entre o manejo baseado na marcha de absorção da cultura e o monitoramento da condutividade elétrica do solo em relação à variável rendimento comercial do tomateiro.

2. O valor da salinidade limiar para o tomateiro Débora Plus cultivado em solo salinizado por fertilizantes foi superior aos valores citados na literatura para solos salinizados por águas salobras.

\section{LITERATURA CITADA}

Alvarenga, M. A. R. Tomate: produção em campo, em casa de vegetação e em hidroponia. 1.ed. Lavras: UFLA, 2004. 400p.

Andriolo, J. L.; Witter, M; Ross, T. dal; Godói, R. S. Crescimento e desenvolvimento do tomateiro cultivado em substrato com reutilização da solução nutritiva drenada. Horticultura Brasileira, v.21, p.485-489, 2003.

Camargo, G. A.; Haj-Isa, N.; Queiroz, M. R. Avaliação da qualidade de tomate seco em conserva. Revista Brasileira de Engenharia Agrícola e Ambiental, v.11, p.521-526, 2007.

Cuartero, J.; Fernandéz-Muñoz, R. Tomato and salinity. Scientia Horticulturae, v.78, p. 83-125, 1999.

Dias, N. da S.; Duarte, S. N.; Gheyi, H. R.; Medeiros, J. F. de; Soares, T. M. Manejo da fertigação e controle da salinidade do solo sob ambiente protegido, utilizando-se extratores de solução do solo. Revista Brasileira de Engenharia Agrícola eAmbiental, v.9, p.496-504, 2005.
Dias, N. S.; Duarte, S. N.; Teles Filho, J. F.; Yoshinaga, R. T. Salinização do solo por aplicação de fertilizantes em ambiente protegido. Irriga, v.12, p.135-143, 2007.

Eloi, W. M.; Duarte, S. N.; Soares, T. M. Níveis de salinidade e manejo da fertigação sobre características do tomateiro cultivado em ambiente protegido. Revista Brasileira de Ciências Agrárias, v.2, p.83-89, 2007.

FAO - Food and Agriculture Organization of the United Nations, FAOSTAT. Database. <http://faostat.fao.org>.27 Fev. 2006.

Furlani, P. R.; Silveira, L. C. P.; Bolonhezi, D.; Faquin, V. Cultivo hidropônico de plantas. Campinas: Instituto Agronômico, 1999. 52p. Boletim Técnico 180

IBGE - Instituto Brasileiro de Geografia e Estatística. Banco de Dados Agregados - SIDRA. 2009. http://www.ibge.gov.br. 19 Fev. 2011.

Maas, E. V.; Hoffman, G. J. Crop salt tolerance - Current assessment. Journal of Irrigation and Drainage Division, v.103, p.115-134, 1977.

Malash, N. M.; Ali, F. A.; Fatahalla, M. A.; Khatabb, E. A.; Hatemb, M. K.; Tawficb, S. Response of tomato to irrigation with saline water applied by different irrigation methods and water management strategies. International Journal of Plant Production, v.2, p101-116, 2008.

Medeiros, P. R. F.; Duarte, S. N.; Dias, C. T. S. Tolerância da cultura do pepino a salinidade em ambiente protegido. Revista Brasileira de Engenharia Agrícola e Ambiental, v.13, p.406-410, 2009.

Nehmi, I. M. D.; Ferraz, J. V.; Nehmi Filho, A. Silva, M. L. Tomate. In: Nehmi, I.M. (coord.). AGRIANUAL 2004: Anuário da agricultura brasileira. São Paulo: FNP - Consultório \& Comércio, 2004. p. 470-478.

Queiroz, S. O. P.; Testezlaf, R.; Matsura, E. E. Metodologia para avaliação da salinidade do solo em ambiente protegido. Irriga, v.14, p.383-397, 2009.

Saranga, Y.; Zamir, D.; Marani, A.; Rudich, J. Breeding tomatoes for salt tolerance: field evaluation of Lycopersicon germplasm for yield and dry-matter production. Journal of the American Society for Horticultural Science, v.116, p.10671071, 1991.

Silva, E. F. F. Manejo da fertigação e controle da salinidade na cultura do pimentão utilizando extratores de solução do solo. Piracicaba: ESALQ/USP, 2002. 136p. Tese Doutorado

Silva, M. O.; Freire, M. B. G. S.; Mendes, A. M.; Freire, F. J.; Sousa, C. E. S.; Góes, G. B. Crescimento de meloeiro e acúmulo de nutrientes na planta sob irrigação com águas salinas. Revista Brasileira de Engenharia Agrícola e Ambiental. v.12, p.593-605, 2008.

Sonneveld, C. La nutrición mineral y salinidad en los cultivos sin suelo: su manejo. In: Gavilán, M. U. (coord.). Tratado de cultivo sin suelo. Madrid Mundi Prensa, 2004. cap. 8, p.305-367. 